\title{
OPTIMAL BOUND FOR THE NUMBER OF (-1)-CURVES ON EXTREMAL RATIONAL SURFACES
}

\author{
MUSTAPHA LAHYANE
}

Received 14 May 2001 and in revised form 12 September 2001

We give an optimal bound for the number of (-1)-curves on an extremal rational surface $X$ under the assumption that $-K_{X}$ is numerically effective and having self-intersection zero. We also prove that a nonelliptic extremal rational surface has at most nine $(-1)$-curves.

2000 Mathematics Subject Classification: 14J26, 14 F05.

1. Introduction. Let $X$ be a smooth projective rational surface defined over the field of complex numbers. From now on we assume that $-K_{X}$ is numerically effective (in short NEF, i.e., the intersection number of the divisor $K_{X}$ with any effective divisor on $X$ is less than or equal to zero, where $K_{X}$ is a canonical divisor on $X$ ) and of selfintersection zero.

It is easy to see that $X$ is obtained by blowing up 9 points (possibly infinitely near) of the projective plane.

Nagata [4] proved that if the 9 points are in general positions, then $X$ has an infinite number of $(-1)$-curves (i.e., smooth rational curves of self-intersection -1 ).

Miranda and Persson [3] studied the case when the position of the 9 points give a rational elliptic surface with a section. They classified all such surfaces which have a finite number of $(-1)$-curves and called them extremal Jacobian elliptic rational surfaces. For each case, they gave the number of $(-1)$-curves.

We use the following notations:

(i) $\sim$ is the linear equivalence of divisors on $X$;

(ii) $[D]$ is the set of divisors $D^{\prime}$ on $X$ such that $D^{\prime} \sim D$;

(iii) $\operatorname{Div}(X)$ is the group of divisors on $X$;

(iv) $N S(X)$ is the quotient group $\operatorname{Div}(X) / \sim$ of $\operatorname{Div}(X)$ by $\sim$ (the linear, algebraic, and numerical equivalences are the same on $\operatorname{Div}(X)$ since $X$ is a rational surface);

(v) $D \cdot D^{\prime}$ denotes the intersection number of the divisor $D$ with the divisor $D^{\prime}$, in particular the self-intersection of $D$ is $D^{2}=D \cdot D$;

(vi) $\bar{D}$ is the element associated to $D$ in $N S(X) \otimes \mathbb{Q}$.

Following [3], we define a smooth rational projective surface having a finite number of $(-1)$-curves on it as an extremal rational surface. The extremal rational surfaces are classified by the following theorem which can be found in [1, Theorem 3.1, page 65].

THEOREM 1.1. Let $X$ be a smooth projective rational surface having $-K_{X} N E F$ and of self-intersection zero. Then the following statements are equivalent: 
(1) $X$ is extremal;

(2) $X$ satisfies the following two conditions:

(a) the rank of the matrix $\left(C_{i} \cdot C_{j}\right)_{i, j=1, \ldots, r}$ is equal to 8 , where $\left\{C_{i}: i=1, \ldots, r\right\}$ is the finite set of (-2)-curves on $X$; a (-2)-curve is a smooth rational curve of self-intersection -2;

(b) there exist $r$ strictly positive rational numbers $a_{i}, i=1, \ldots, r$, such that $-\bar{K}_{X}=\sum_{i=1}^{i=r} a_{i} \bar{C}_{i}$.

From this theorem we deduce the following lemma.

LEMmA 1.2. Let $X$ be an extremal surface. With the same notation as Theorem 1.1, if all of the $a_{i}, i=1, \ldots, r$, are strictly positive integers, then $a(-1)$-curve on $X$ meets only one (-2)-curve $C_{i}$ in one point and necessarily the coefficient $a_{i}$ of $C_{i}$ must be equal to one.

Proof. Let $E$ be a $(-1)$-curve on $X$. We have $\sum_{i=1}^{i=r} a_{i} E \cdot C_{i}=1$ (since $-\bar{K}_{X}=\sum_{i=1}^{i=r} a_{i} \bar{C}_{i}$ and $E$ is a (-1)-curve). On the other hand, for every $j \in\{1,2, \ldots, r\}$, the intersection number of $E$ with $C_{j}$ is a nonnegative integer. Therefore, there exists $i \in\{1,2, \ldots, r\}$ such that $a_{i} E \cdot C_{i}=1$ and for every $j \in\{1,2, \ldots, r\}, j \neq i, E \cdot C_{j}=0$. Hence the lemma follows.

In this note, we give an optimal bound for the number of $(-1)$-curves on an extremal rational surface. Keeping the same notations as in Theorem 1.1, our result is as follows.

THEOREM 1.3. Let $X$ be an extremal rational surface. The number of (-1)-curves on $X$ is bounded by the integer

$$
-1+\prod_{i=1}^{i=r}\left(1+\left[\left[\frac{1}{a_{i}}\right]\right]\right),
$$

where [ [ ] denotes the greatest integer function. This bound is optimal.

2. The proof. Let $X$ be a smooth projective rational surface such that $K_{X}^{2}=0$, where $K_{X}$ is a canonical divisor of $X$. We assume that $-K_{X}$ is NEF, that is, $K_{X} \cdot D \leq 0$ for every effective divisor $D$ on $X$.

For each $(r+2)$-tuple $\left(p, q ; n_{1}, \ldots, n_{r}\right)$ of integers, where $r$ is a strictly positive integer, we consider the set $\mathscr{E}_{p, q}^{n_{1}, \ldots, n_{r}}$ of divisor classes [D] on $X$ such that

(i) $D^{2}=p$,

(ii) $D \cdot K_{X}=q$,

(iii) $D \cdot C_{i}=n_{i}$ for each $i=1, \ldots, r$, where $\left\{C_{i}: i=1, \ldots, r\right\}$ is the finite set of $(-2)$ curves on $X$.

We think of $\mathscr{C}_{0} n_{1}, \ldots, n_{r}$ as a set of elements of $N S(X)$ with imposed intersection with the set of $(-2)$-curves like a linear system with imposed base points. We prove that if the set of $(-2)$-curves on $X$ is maximal in a sense that will be explained in Proposition 2.1, then for each nonzero integer $\mathcal{q}$, the set $\mathscr{E}_{p, q}^{n_{1}, \ldots, n_{r}}$ has at most one element. 
Proposition 2.1. Let $X$ be a smooth projective rational surface having an anticanonical divisor $-K_{X}$ of self-intersection zero. If the set of $(-2)$-curves on $X$ spans the orthogonal complement of $K_{X}$, then for each $(r+2)$-tuple $\left(p, q ; n_{1}, \ldots, n_{r}\right)$ of integers, with $q$ nonzero, the set $\mathscr{C}_{p, q}^{n_{1}, \ldots, n_{r}}$ has at most one element.

Proof. If the set $\mathscr{E}_{p, q}^{n_{1}, \ldots, n_{r}}$ is not empty, consider two elements $[D]$ and $\left[D^{\prime}\right]$. First, we have $D-D^{\prime}$ belongs to the orthogonal complement of $K_{X}$ since $D \cdot K_{X}=q=D^{\prime} \cdot K_{X}$, keeping in mind that $D-D^{\prime}$ is orthogonal to each $C_{i}$, for $i=1, \ldots, r$, (since $D \cdot C_{i}=$ $D^{\prime} \cdot C_{i}$ for each $\left.i=i=1, \ldots, r\right)$ and the fact that the set of $(-2)$-curves on $X$ spans the orthogonal complement of $K_{X}$, we conclude that $\left(D-D^{\prime}\right)^{2}=0$. Hence there exists a rational number $m$ such that $\bar{D}=\overline{D^{\prime}}+m \overline{K_{X}}$. Furthermore $D^{2}=D^{\prime 2}$. Since $q \neq 0$, we have $m=0$ and hence $D$ is linearly equivalent to $D^{\prime}$, that is, $[D]=\left[D^{\prime}\right]$.

An immediate consequence is the following corollary.

COROLLARY 2.2. Let $X$ be a smooth projective rational surface having an anticanonical divisor $-K_{X}$ of self-intersection zero. If the set of $(-2)$-curves on $X$ spans the orthogonal complement of $K_{X}$, then for two different $(-1)$-curves $E$ and $E^{\prime}$ on $X$, there exists $i \in\{1, \ldots, r\}$ such that $C_{i} \cdot E \neq C_{i} \cdot E^{\prime}$, where $\left\{C_{1}, \ldots, C_{r}\right\}$ is the set of $(-2)$-curves on $X$.

Proof of Theorem 1.3. Let $E$ be a $(-1)$-curve on $X$. From Theorem 1.1(2)(b), we have $0 \leq E \cdot C_{i} \leq\left[\left[1 / a_{i}\right]\right]$ for each $i=1, \ldots, r$. The fact that $E \cdot\left(-K_{X}\right)=1$ implies that there exists $j_{E} \in\{1, \ldots, r\}$ such that $E \cdot C_{j_{E}} \geq 1$, so the $r$-tuple $\left(E \cdot C_{i}\right)_{i=1, \ldots, r}$ of integers belongs to the set $\prod_{i=1}^{i=r}\left(\left[0,\left[\left[1 / a_{i}\right]\right]\right] \cap \mathbb{N}\right) \backslash\{(0, \ldots, 0)\}$ which has exactly $-1+\prod_{i=1}^{i=r}\left(1+\left[\left[1 / a_{i}\right]\right]\right)$ elements. Consider the map $\phi$ defined from the set of $(-1)$ curves on $X$ to $\prod_{i=1}^{i=r}\left(\left[0,\left[\left[1 / a_{i}\right]\right]\right] \cap \mathbb{N}\right) \backslash\{(0, \ldots, 0)\}$, it is given by $\phi(E)=\left(E \cdot C_{i}\right)_{i=1, \ldots, r}$ for every $(-1)$-curve $E$ on $X$. Corollary 2.2 confirm that $\phi$ is injective. Therefore, the first result of Theorem 1.3 holds.

The suggested bound is optimal for certain extremal rational surfaces (see Remark 2.3).

REMARK 2.3. It is interesting to know that for which extremal rational surfaces, the set of $(-1)$-curves is in one-to-one correspondence with $\prod_{i=1}^{i=r}\left(\left[0,\left[\left[1 / a_{i}\right]\right]\right] \cap \mathbb{N}\right) \backslash\{(0, \ldots$, $0)$ \}. For example, in the case of an extremal elliptic Jacobian rational surface [3, Table 5.1, page 544], the only such surfaces for which there is a bijection are

(i) the surface $X_{22}$ which has the set $\left\{I I, I I^{*}\right\}$ as set of singular fibers;

(ii) the surface $X_{211}$ which has the set $\left\{I I^{*}, I_{1}, I_{1}\right\}$ as set of singular fibers.

More generally, for a given extremal surface $X$, we ask: which $r$-tuple $\left(n_{1}, \ldots, n_{r}\right)$ of $\prod_{i=1}^{i=r}\left(\left[0,\left[\left[1 / a_{i}\right]\right]\right] \cap \mathbb{N}\right) \backslash\{(0, \ldots, 0)\}$ represent a $(-1)$-curve? Very little is known about this question.

REMARK 2.4. Let $X$ be an extremal rational surface which is not elliptic, then we have the following facts:

(1) the set of (-2)-curves on $X$ is connected and hence has one of the three types of configurations $\tilde{A}_{8}, \tilde{D}_{8}$, or $\tilde{E}_{8}$. In all cases there are only nine (-2)-curves on the surface; 
(2) $\overline{-K_{X}}$ can only be written in one manner as strictly positive linear combination of the nine $(-2)$-curves.

In fact these properties are consequences of the following two facts:

(1) if zero is a nontrivial linear combination of the set of $(-2)$-curves, then the surface must be elliptic (see [1, Proposition 1.2, page 26]);

(2) if a divisor is orthogonal to $K_{X}$ and of self-intersection zero, then it is a multiple of $K_{X}$ (see [2, Lemma 2]).

Now we consider examples of surfaces with different configurations of (-2)-curves.

CASE 1 (the configuration is $\tilde{E}_{8}$ ). We have

$$
-K_{X}=C_{1}+2 C_{2}+3 C_{3}+4 C_{4}+5 C_{5}+6 C_{6}+4 C_{7}+2 C_{8}+3 C_{9} .
$$

Our bound is equal to 1 , consequently there is only one $(-1)$-curve: the exceptional divisor of the last blowup.

CASE 2 (the configuration is $\tilde{D}_{8}$ ). We have

$$
-K_{X}=C_{1}+C_{2}+2 C_{3}+2 C_{4}+2 C_{5}+2 C_{6}+2 C_{7}+C_{8}+C_{9} .
$$

Using Lemma 1.2 and Corollary 2.2, we deduce that the number of $(-1)$-curves is at most 4 , whereas our bound is 15 .

CASE 3 (the configuration is $\tilde{A}_{8}$ ). We have

$$
-K_{X}=C_{1}+C_{2}+C_{3}+C_{4}+C_{5}+C_{6}+C_{7}+C_{8}+C_{9} .
$$

Using Lemma 1.2 and Corollary 2.2, we deduce that the number of $(-1)$-curves is at most 9, whereas our bound is 255 .

ACKNOWLEDGments. I would like to thank Professor Peter Russell for many useful discussions and for his hospitality when I was in the nice Mathematics Department of McGill University. Many thanks also to Abdus Salam International Centre for Theoretical Physics, to Departamento de Álgebra, Geometría y Topología, Universidad de Valladolid.

\section{REFERENCES}

[1] M. Lahyane, Courbes Exceptionnelles sur les Surfaces Rationnelles avec $K^{2}=0$, Ph.D. thesis, Université de Nice Sophia-Antipolis, Nice, France, 1998.

[2] __ Irreducibility of the (-1)-classes on smooth rational surfaces, preprint of the Abdus Salam ICTP, Trieste, Italy, 2001.

[3] R. Miranda and U. Persson, On extremal rational elliptic surfaces, Math. Z. 193 (1986), no. 4, 537-558.

[4] M. Nagata, On rational surfaces. II, Mem. Coll. Sci. Univ. Kyoto Ser. A Math. 33 (1960), 271-293.

Mustapha lahyane: Abdus Salam International Centre for Theoretical Physics, TRIESTE 34100, ITALY

E-mail address: 1ahyanem@ictp.trieste.it 


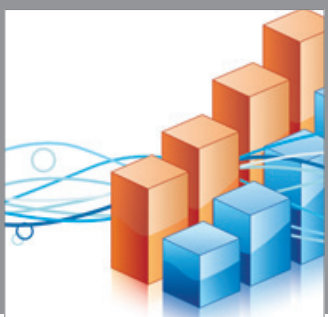

Advances in

Operations Research

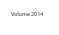

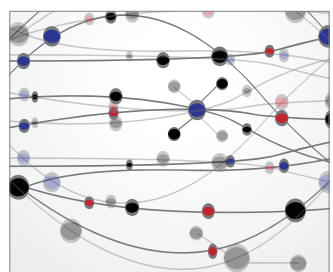

\section{The Scientific} World Journal
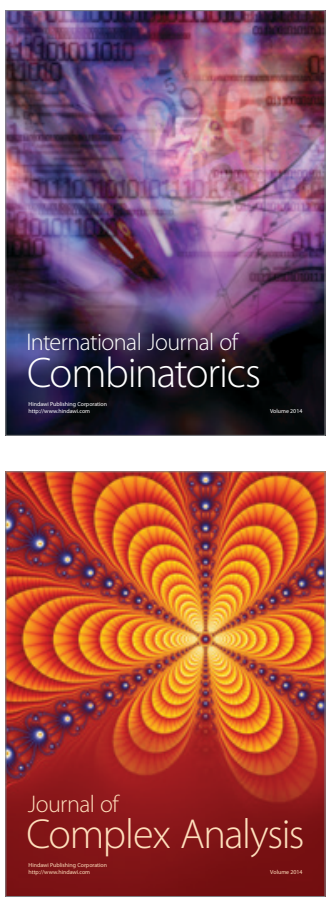

International Journal of

Mathematics and

Mathematical

Sciences
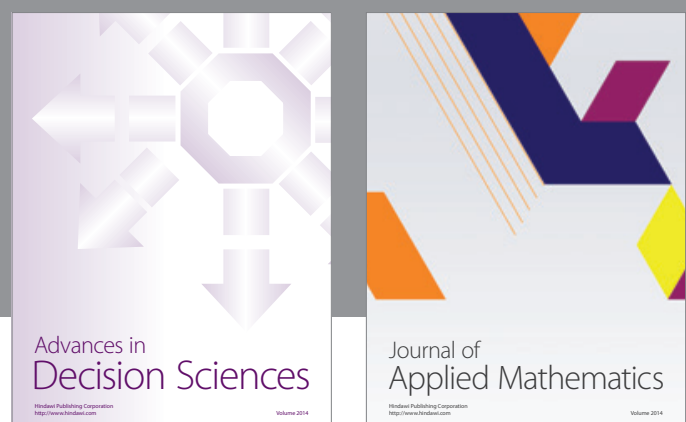

Journal of

Applied Mathematics
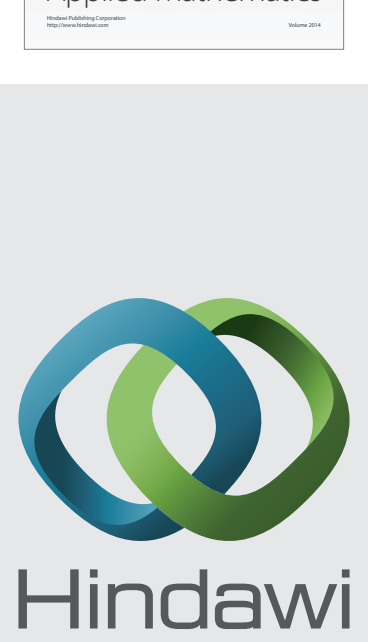

Submit your manuscripts at http://www.hindawi.com
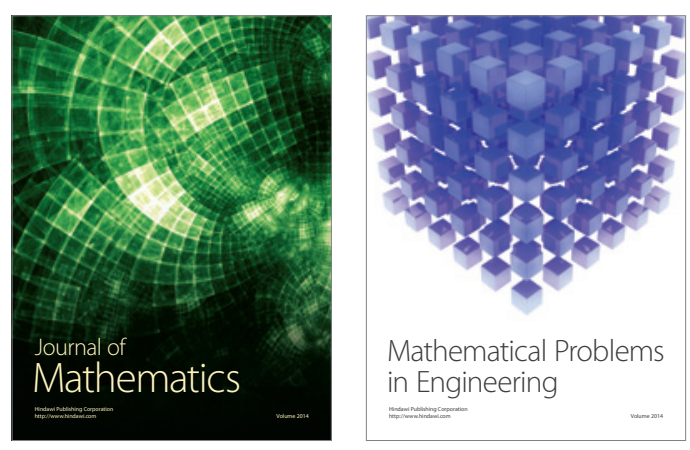

Mathematical Problems in Engineering
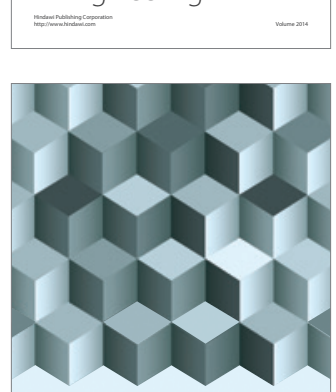

Journal of

Function Spaces
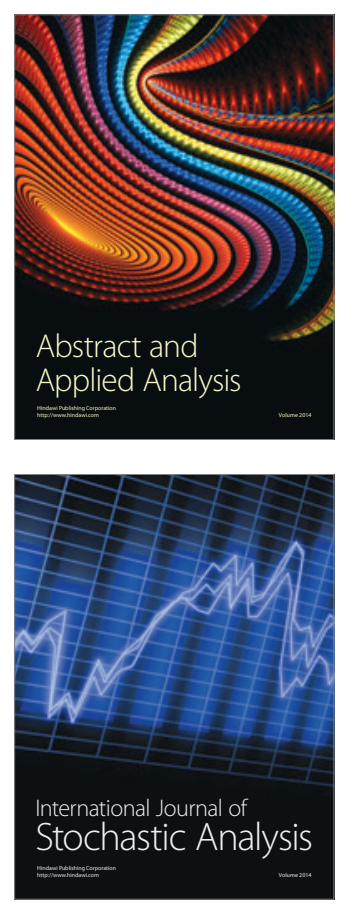

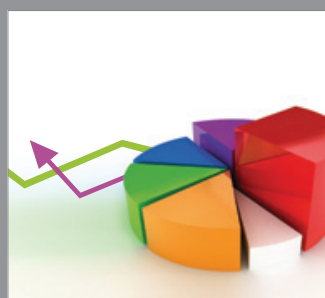

ournal of

Probability and Statistics

Promensencen
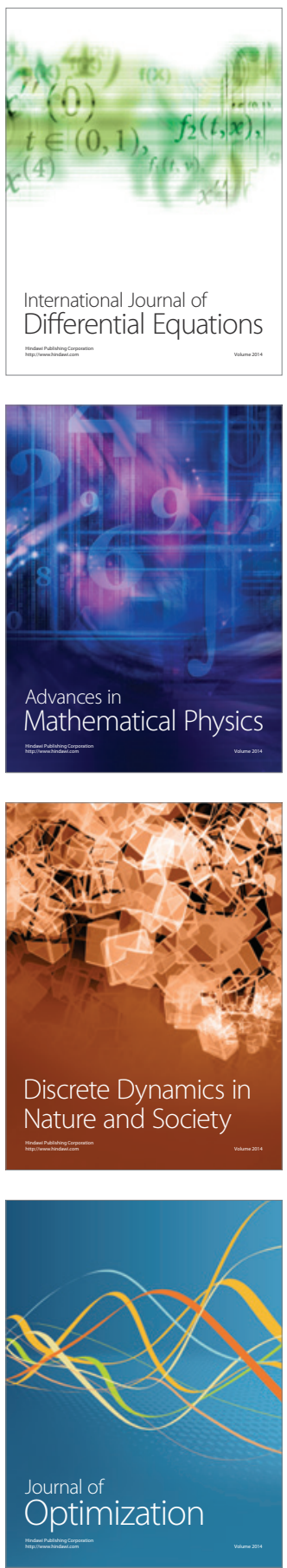